\title{
Non-minimum phase identification based on higher order spectrum slices
}

\author{
Amar Kachenoura ${ }^{(1,2)}$, student member, IEEE, Laurent Albera ${ }^{(1,2)}$, member, IEEE, Jean-Jacques \\ Bellanger $^{(1,2)}$, Lotfi Senhadji ${ }^{(1,2)}$, senior member, IEEE \\ 1 INSERM, U642, Rennes, F-35000, France; \\ 2 Université de Rennes 1, LTSI, Rennes, F-35000, France. \\ For Correspondence : Amar Kachenoura \\ Tel : (33) - $223235601 / 6741716$ 91, E-Mail : amar.kachenoura@univ-rennes1.fr
}

\begin{abstract}
A new family of methods, named PEP (Phase Estimation using Polyspectrum slices), for the reconstruction of the Fourier phase of a complex LTI system excited by a white non-Gaussian input is proposed. More precisely, we propose two subfamilies of methods, the $q$-PEP $(q \geq 3)$ and $\left(q_{1}, q_{2}\right)$-PEP $\left(q_{2}>q_{1} \geq 3\right)$ algorithms. The $q$-PEP methods exploit the best Two-Dimensional (2D) slice of the data $q$-th order spectrum. The originality of the $\left(q_{1}, q_{2}\right)$-PEP methods consists of exploiting simultaneously one 1D slice of the $q_{1}$-th order spectrum and one 2D slice of the $q_{2}$-th order spectrum. These new algorithms are easy both to implement and to use. Moreover, the asymptotic unbiasedness and consistency of these methods are demonstrated. Eventually, computer simulations show that the PEP algorithms exhibit in general better performances than classical methods especially for band-limited systems.
\end{abstract}

\section{INTRODUCTION}

System reconstruction and especially phase recovery is a significant problem which arises in many applications, such as data communications [2], seismic, speech processing, medical diagnosis and other deconvolution problems [5]. More particularly, in astronomy, high resolution imaging from groundbased telescopes involves a phase recovery to overcome the severe atmospheric degradation [12].

Because physical systems are mostly non-minimum phase, we consider, in our paper, the phase retrieval problem of a nonminimum phase Linear Time Invariant (LTI) system excited by an i.i.d. (independently and identically distributed), zero mean and non-Gaussian input. In such a context, the phase recovery of a non-minimum phase system can be achieved from the output using higher order spectra (polyspectra). Indeed, in opposition to the power spectrum which preserves only the magnitude information of systems, the polyspectra also preserve the true phase character of systems. Several methods for phase reconstruction using polyspectra have been developed (see [15] [16] for a review). These methods can be divided in two subcategories: those that use the whole $q$-th order ( $q \geq 3$ ) spectrum information [3] [1] [13] [17] [19] and those that use only some part of this information such as one or two fixed One-Dimensional (1D) polyspectrum slices [10] [7] [20] [21]. The latter algorithms assume that there is a criterion for selecting the most useful polyspectrum region providing a measure of slice goodness. The algorithm presented in [21] proposes such a procedure, named the 1D frequency content. This selection procedure potentially enables us to avoid regions where polyspectrum estimates exhibit high variance or regions where the ideal polyspectrum is expected to be zero, as in the case of band-limited systems. Another way to describe and to differentiate algorithms named before, consists of classifying them according to their recursive or non-recursive nature. Indeed, the algorithms [3] [1] [10] [20] are recursive in nature: they calculate, sequentially, the phase values under the assumption that the first value is equal to zero. Methods proposed in [13] [7] [17] [19] [21] are not recursive: they estimate all phase values at the same time. Eventually, methods presented in [3] [13] [7] [17] [19] require an additional step of phase unwrapping, in contrast to those introduced in [1] [10] [20] [21].

Each of these methods present some limitations. Methods which use the whole polyspectrum information [3] [1] [13] [17] [19] are generally less effective on systems which have a band-limited frequency response as shown in [21]. Besides, Rangoussi et al. [17] and Lii et al. [10] have developed algorithms which are only valid for real systems. In addition, the method given in [20] does not allow for handling a linear system whose input sequence is symmetrically distributed. It should be noted that since methods [3] [1] [10] [20] are recursive in nature, phase estimation errors in the lowfrequency samples can propagate to high-frequency samples as well. Finally, computer results presented in [20] [21] show that the performances of algorithms proposed in [20] [21] depend on the selected 1D polyspectrum slice.

In order to overcome the limitations of the previous algorithms, a new family of phase retrieval methods, based on multiple higher order spectrum slices, named PEP (Phase Estimation using Polyspectrum slices), is proposed. A panel of algorithms which are easy to use and to implement is provided. These algorithms can handle any kind of non-minimum phase SISO (Single Input Single output) systems, they are not recursive and do not estimate all the phase values at the same time. They estimate each phase value independently from the others. More precisely, we propose two subfamilies of methods, the $q$-PEP $(q \geq 3)$ and $\left(q_{1}, q_{2}\right)$-PEP $\left(q_{2}>q_{1} \geq 3\right)$ algorithms. 
The $q$-PEP methods exploit the best Two-Dimensional (2D) slice of the data $q$-th order spectrum. The originality of the $\left(q_{1}, q_{2}\right)$-PEP methods is the simultaneous exploitation of one 1D slice of the $q_{1}$-th order spectrum and one 2D slice of the $q_{2}$-th order spectrum. This joint exploitation of two distinct $q$ th order spectra allows for a better processing of band-limited systems. Computer results show that the $\left(q_{1}, q_{2}\right)$-PEP methods are less sensitive to a wrong selection of the best polyspectrum slice. Finally, note that all the proposed methods require an additional phase unwrapping step.

The paper is organized as follows: in section II, the phase recovery problem is stated and the definition of the $q$-th $(q>$ 2 ) order spectra is recalled. In section III, the PEP approach is presented and its asymptotic consistency analysis is provided. Section V gives computer results of some PEP algorithms and classical methods, and a conclusion is given in section VI.

\section{Problem FORMulation AND STATISTICS}

\section{A. Problem statement}

It is assumed throughout the paper that $M$ complex samples of a discrete random process $\{x(m)\}_{m \in \mathbb{Z}}$ are observed, and that each random variable $x(m)$ satisfies the following LTI model:

$$
x(m)=\sum_{\ell \in \mathbb{Z}} h(\ell) s(m-\ell)+\nu(m)
$$

where $\{s(m)\}_{m \in \mathbb{Z}}$ and $\{\nu(m)\}_{m \in \mathbb{Z}}$ represent the input and additive noise sequences, respectively, and where:

$$
h(m) \stackrel{\text { def }}{=} \frac{1}{2 \pi} \int_{-\pi}^{+\pi} \mathcal{H}(\omega) \mathrm{e}^{\mathrm{i} \omega m} d \omega
$$

is the $m$-th tap of the LTI system whose frequency response is $\mathcal{H}$. The problem dealt with in this paper can be formulated as following:

Problem 1: Let $\mathcal{H}(\omega)=|\mathcal{H}(\omega)| \mathrm{e}^{\mathrm{i} \phi_{h}(\omega)}$ be the frequency response at point $\omega$ of the considered non-minimum phase LTI system, find the phase response, $\phi_{h}$, of the system only

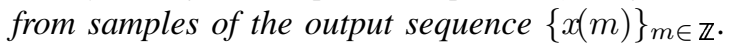

The following assumptions are then placed on the system and the signals involved in order to solve problem 1 .

(A1) $\{s(m)\}_{m \in \mathbb{Z}}$ is an i.i.d. non-gaussian, stationary and ergodic complex sequence;

(A2) $\{\nu(m)\}_{m \in \mathbb{Z}}$ is stationary, ergodic, gaussian with components in the complex field, and independent of the input source;

(A3) All $q$-th $(q>2)$ order marginal source cumulants are absolutely summable and all $q$-th order spectra are non-zero in the frequency band over which the channel response is non-zero (higher order cumulants and polyspectra will be described hereafter);

(A4) The LTI system is stable (i.e. $\{h(m)\}_{m \in \mathbb{Z}}$ is absolutely summable, which guarantees the existence of a bounded frequency response) with complex taps.

It is noteworthy that a unique solution of problem 1 can be found only up to a linear phase. Indeed, as shown in equation (1), the expression of $x(m)$ does not change when we replace $\ell$ by $\ell^{\prime}+\tau$ where $\tau$ is a delay or when we multiply the impulse response and the input source by a complex exponential and its conjugate, respectively.

\section{B. Cumulants and polyspectra}

Let $C_{r, x}^{q-r}\left(m, \tau_{1}, \ldots, \tau_{q-1}\right)=\operatorname{Cum}\left\{x(m), x\left(m+\tau_{1}\right), \ldots\right.$ $\left., x\left(m+\tau_{r-1}\right), x\left(m+\tau_{r}\right)^{*}, \ldots, x\left(m+\tau_{q}\right)^{*}\right\}$ be the $q$-th $(q \geq 3)$ order cumulant [14] of $x(m)$, where $r$ terms are not conjugated and $q-r$ terms are conjugated. Under assumption (A1) and (A2), process $\{x(m)\}_{m \in \mathbb{Z}}$ is stationary. Consequently, it's $q$ th order cumulants do not depend on time $m$ and can be denoted by $C_{r, x}^{q-r}\left(\tau_{1}, \ldots, \tau_{q-1}\right)$. Then, under assumption (A3), it is possible to define the $q$-th order spectrum as following [15]:

Definition 1: The q-th order spectrum is given by the (q1)-dimensional Discrete Fourier Transform (DFT) of the q-th order cumulant.

Using assumptions (A1), (A2) and the multilinearity property shared by all moments and cumulants [14], the $q$-th order spectrum of the output data can be related to the $q$-th order marginal source cumulant, as shown by property 1 :

Property 1: Let $\{x(m)\}_{m \in \mathbb{Z}}$ be the discrete random process given by (1). Its $q$-th order spectrum satisfies the following equality:

$$
\begin{aligned}
\Gamma_{r, x}^{q-r}\left(\omega_{1}, \ldots, \omega_{q-1}\right)= & C_{r, s}^{q-r} \mathcal{H}\left(-\omega_{1}-\ldots-\omega_{q-1}\right) \mathcal{H}\left(\omega_{1}\right) \\
& \mathcal{H}\left(\omega_{r-1}\right) \mathcal{H}\left(-\omega_{r}\right)^{*} \ldots \mathcal{H}\left(-\omega_{q-1}\right)^{*}
\end{aligned}
$$

where $C_{r, s}^{q-r} \stackrel{\text { def }}{=} C_{r, s}^{q-r}(0, \ldots, 0)$ and $\mathcal{H}$ denote the $q$-th order marginal source cumulant associated with null delays and the system frequency response, respectively.

Note that, in practical contexts, cumulants and spectra cannot be exactly computed and they have to be estimated from data samples using some estimation procedures [14] [15].

\section{ALgORITHMS}

\section{A. The $q$-PEP methods $(q \geq 3)$}

This approach exploits one 2D slice of the output data $q$-th order spectrum $\Gamma_{r, x}^{q-r}$ of the output sequence, that is, the matrix extracted from $\Gamma_{r, x}^{q-r}$ by fixing $q-3$ frequencies and varying the two latter frequencies between $\pm \pi$. Although the 3-PEP method was briefly presented in [9] and since an extension to $q$-th order $(q>4)$ spectra is straightforward, we only illustrate the $q$-PEP method for $q=4$.

Let $\psi_{2, x}^{2}$ be the phase of the data trispectrum $\Gamma_{2, x}^{2}$. Moreover, we will consider discrete frequencies in the sequel, i.e., $\omega_{j}=(2 \pi / N) k_{j}$ with $k_{j} \in\{0, \ldots, N-1\}$ and $j \in\{1, \ldots, q-$ $1\}$. By omitting the factor $2 \pi / N$, the relationship between the phases of the quantities involved in $(3)$, for $(q, r)=(4,2)$, is given by:

$$
\begin{aligned}
\psi_{2, x}^{2}\left(k_{1}, k_{2}, k_{3}\right)= & \xi_{2, s}^{2}+\phi_{h}\left(-k_{1}-k_{2}-k_{3}\right)+ \\
& \phi_{h}\left(k_{1}\right)-\phi_{h}\left(-k_{2}\right)-\phi_{h}\left(-k_{3}\right)
\end{aligned}
$$

where $\xi_{2, s}^{2}$ is the phase of the marginal source cumulant $C_{2, s}^{2}$. Note that $\xi_{2, s}^{2}$ is a multiple of $\pi$ since $C_{2, s}^{2}$ is necessarily a real 
number. Let $k_{3}$ be fixed to an integer, $\alpha \in\{0,1, \ldots, N-1\}$, using a simple measure of 2D slice goodness which will be presented in section III-C. Because the $2 \pi$-periodicity of $\mathcal{H}$ implies the $N$-periodicity of its discrete phase $\phi_{h}$, the sum of $\psi_{2, x}^{2}(., ., \alpha)$ over the discrete frequencies $k_{2}\left(0 \leq k_{2}<N\right)$ is given for each discrete frequency $k_{1}\left(0 \leq k_{1}<N\right)$ by:

$$
\sum_{k_{2}=0}^{N-1} \psi_{2, x}^{2}\left(k_{1}, k_{2}, \alpha\right)=N\left(\phi_{h}\left(k_{1}\right)+\xi_{2, s}^{2}-\phi_{h}(-\alpha)\right)
$$

Although equation (5) seems to provide a solution for the estimation of $\phi_{h}$ from $\psi_{2 x}^{2}$, it is not a convenient formula for phase retrieval. Indeed, the trispectrum phase $\psi_{2, x}^{2}$ is generally estimated by its principal value, $\tilde{\psi}_{2}^{2}$, given, for every $k_{1}, k_{2}, k_{3}\left(0 \leq k_{1}, k_{2}, k_{3}<N\right)$, by:

$$
\begin{aligned}
& \tilde{\psi}_{2, x}^{2}\left(k_{1}, k_{2}, k_{3}\right)= \\
& \quad \arctan \left(\Im\left(\Gamma_{2, x}^{2}\left(k_{1}, k_{2}, k_{3}\right)\right), \Re\left(\Gamma_{2, x}^{2}\left(k_{1}, k_{2}, k_{3}\right)\right)\right)
\end{aligned}
$$

where $\Re$ and $\Im$ refer to as the real and imaginary parts, and arctan is the four-quadrant arc tangent operator that forces the angle function $\tilde{\psi}_{2 x}^{2}$ to lie between $\pm \pi$ radians. These principal values are also called wrapped phase values because the absolute phase is wrapped into the interval $[-\pi, \pi]$ by the following non-linear process:

$$
\begin{aligned}
& \forall\left(k_{1}, k_{2}, k_{3}\right) \in\{0,1, \ldots, N-1\}^{3} \\
& \quad \tilde{\psi}_{2, x}^{2}\left(k_{1}, k_{2}, k_{3}\right)=\psi_{2, x}^{2}\left(k_{1}, k_{2}, k_{3}\right)+2 \pi I\left(k_{1}, k_{2}, k_{3}\right)
\end{aligned}
$$

where $I$ is an integer function that forces $\tilde{\psi}_{2}^{2}$ to belong to $[-\pi, \pi]$. Thus, fixing $k_{3}$ to $\alpha$ in (7), summing the result over the discrete frequencies $k_{2}\left(0 \leq k_{2}<N\right)$ and using (5), we have for every $k_{1}\left(0 \leq k_{1}<N\right)$ :

$$
\begin{array}{r}
\sum_{k_{2}=0}^{N-1} \tilde{\psi}_{2, x}^{2}\left(k_{1}, k_{2}, \alpha\right)=N\left(\phi_{h}\left(k_{1}\right)+\xi_{2, s}^{2}-\phi_{h}(-\alpha)\right) \\
+2 \pi \sum_{k_{2}=0}^{N-1} I\left(k_{1}, k_{2}, \alpha\right)
\end{array}
$$

The discrete phase function $\phi_{h}$ can thus be extracted from equation (8) provided that a phase unwrapping scheme is performed before extraction. In other words, the following phase unwrapping problem has to be solved:

Problem 2: Let $q$ and $\tilde{\psi}$ be a non-zero integer and a phase function of $\{0,1, \ldots, N-1\}^{q}$ into $[-\pi, \pi]$, respectively, find the phase jump function $J$ of $\{0,1, \ldots, N-1\}^{q}$ into $\mathbb{Z}$ such that the unwrapped phase function $\tilde{\psi}^{u}$ given by:

$$
\forall \boldsymbol{k} \in\{0,1, \ldots, N-1\}^{q}, \quad \tilde{\psi}^{u}(\boldsymbol{k})=\tilde{\psi}(\boldsymbol{k})+2 \pi J(\boldsymbol{k})
$$

is as continuous (smooth) as possible.

Some methods can be found in the literature in order to solve problem 2 (see [8] and [6] for $q=2$ and $q=3$, respectively). However, note that problem 2 has not a unique solution, but it has a class of solutions, pairwise equal within a multiple of $2 \pi$. Consequently, in the light of equation (8), the unwrapping step can be achieved at three different levels, each one leading to a particular phase retrieval approach. The first approach consists in applying a Three-Dimensional (3D) phase unwrapping scheme [6] to $\tilde{\psi}_{2, x}^{2}$ to obtain, for every $k_{1}, k_{2}, k_{3}$ $\left(0 \leq k_{1}, k_{2}, k_{3}<N\right)$, an estimate, $\tilde{\psi}_{2 x}^{2, u}$, of $\psi_{2, x}^{2}$ up to an additive constant:

$$
\tilde{\psi}_{2, x}^{2, u}\left(k_{1}, k_{2}, k_{3}\right)=\psi_{2, x}^{2}\left(k_{1}, k_{2}, k_{3}\right)+2 \pi I_{u}
$$

where $I_{u}$ is an unknown integer constant. An estimate, $\tilde{\phi}_{h}$, of $\phi_{h}$ up to an additive constant, $a(\alpha)=\xi_{2}^{2} s-\phi(-\alpha)+2 \pi I_{u}$, may then be derived by i) fixing $k_{3}$ to $\alpha$, ii) summing over $k_{2}$ and iii) dividing by $N$. A second solution can be obtained using a 2D phase unwrapping process [8] of the function $\tilde{\psi}_{2, x}^{2}(., ., \alpha)$ where $\alpha$ is fixed. Then, we get, for every $k_{1}, k_{2}\left(0 \leq k_{1}, k_{2}<\right.$ $N)$, an estimate, $\tilde{\psi}_{2, x}^{2 u}(., ., \alpha)$ of $\psi_{2, x}^{2}(., ., \alpha)$ up to an additive constant:

$$
\tilde{\psi}_{2, x}^{2, u}\left(k_{1}, k_{2}, \alpha\right)=\psi_{2, x}^{2}\left(k_{1}, k_{2}, \alpha\right)+2 \pi I_{u}^{\prime}(\alpha)
$$

where $I_{u}^{\prime}(\alpha)$ is an unknown integer constant. An estimate, $\tilde{\phi}_{h}$, of $\phi_{h}$ can thus be derived, from (11), using the following equation, for every $k_{1}\left(0 \leq k_{1}<N\right)$ :

$$
\tilde{\phi}_{h}\left(k_{1}\right) \stackrel{\text { def }}{=} \frac{1}{N} \sum_{k_{2}=0}^{N-1} \tilde{\psi}_{2, x}^{2, u}\left(k_{1}, k_{2}, \alpha\right)=\phi_{h}\left(k_{1}\right)+a^{\prime}(\alpha)
$$

where the constant $a^{\prime}(\alpha)$ is given by $a^{\prime}(\alpha)=\xi_{2, s}^{2}-\phi(-\alpha)+$ $2 \pi I_{u}^{\prime}(\alpha)$. The third and last approach consists of applying a $1 \mathrm{D}$ phase unwrapping procedure to the left term of equation (8) and dividing the result by $N$. We then obtain a new estimate, $\tilde{\phi}_{h}$, of $\phi_{h}$ up to an additive constant $a^{\prime \prime}(\alpha)=\xi_{2, s}^{2}-$ $\phi(-\alpha)+2 \pi I^{\prime \prime}(\alpha) / N$ where $I^{\prime \prime}(\alpha)$ is an unknown integer. For the sake of convenience, the three 4-PEP approaches presented previously will be referred, in the sequel, to as $4-\mathrm{PEP}_{3 \mathrm{D}}, 4-$ $\mathrm{PEP}_{2 \mathrm{D}}$ and $4-\mathrm{PEP}_{1 \mathrm{D}}$, respectively. Besides, since a $q$-th order spectrum $(q>3)$ may contain several 2D slices of sufficient goodness, an improved final phase estimate can be obtained by averaging. In such a case, the averaging can be done in the $\mathrm{e}^{\mathrm{i} \cdot}$ domain just before the division by $N$. Moreover, if the filter impulse response has taps with values in the complex field, a non-zero value has to be chosen for $\alpha$. Indeed, if $\alpha$ is nul then equation (5) shows that $\phi_{h}(0)$ cannot be estimated. On the other hand, when the system is real, $\phi_{h}(0)$ is equal to zero. Consequently, $\alpha$ can be set to zero and the constants $a(0), a^{\prime}(0)$ or $a^{\prime \prime}(0)$ can be deduced from $\tilde{\phi}_{h}(0)$. Note that for real systems, the constants $a(0)$ and $a^{\prime}(0)$ are necessarily multiples of $\pi$ since $\xi_{2, s}^{2}$ is a multiple of $\pi$.

\section{B. The $\left(q_{1}, q_{2}\right)$-PEP methods $\left(q_{2}>q_{1} \geq 3\right)$}

The originality of this algorithm is the joint exploitation of a 1D slice of one $q_{1}$-th order spectrum and one $2 \mathrm{D}$ slice of the $q_{2}$-th order spectrum $\left(q_{2}>q_{1} \geq 3\right)$. As an example, the approach is presented hereafter using the fourth $\left(q_{1}=4\right)$ and sixth $\left(q_{2}=6\right)$ order spectra, named trispectrum and quintuspectrum of the data respectively. The method using the third and the fourth orders was briefly investigated in [9]. The extension to the $\left(q_{1}, q_{2}\right)$-th order, such as $\left(q_{1}, q_{2}\right)=(4,5)$ or $q_{2}>q_{1} \geq 5$, can be easily realized from the following discussion.

For $\left(q_{2}, r_{2}\right)=(6,3)$, property 1 implies, for every $k_{1}, k_{2}, k_{3}, k_{4}, k_{5}\left(0 \leq k_{1}, k_{2}, k_{3}, k_{4}, k_{5}<N\right)$ :

$$
\begin{gathered}
\psi_{3, x}^{3}\left(k_{1}, k_{5}, k_{3}, k_{4}, k_{2}\right)=\phi_{h}\left(-k_{1}-k_{5}-k_{3}-k_{4}-k_{2}\right)+ \\
\phi_{h}\left(k_{1}\right)+\phi_{h}\left(k_{5}\right)-\phi_{h}\left(-k_{3}\right)-\phi_{h}\left(-k_{4}\right)-\phi_{h}\left(-k_{2}\right)+\xi_{3, s}^{3}
\end{gathered}
$$


where $\psi_{3, x}^{3}$ is the phase of the discrete output quintuspectrum $\Gamma_{3, x}^{3}$ and $\xi_{3, s}^{3}$ is the phase associated with the marginal source cumulant $C_{3, s}^{3}$. According to the cumulant definition, it appears that $C_{3, s}^{3}$ is a real number and thus $\xi_{3, s}^{3}$ is a multiple of $\pi$. Based on the difference between equations (13) and (4), we get:

$$
\begin{array}{r}
\psi_{3, x}^{3}\left(k_{1}, k_{5}, k_{3}, k_{4}, k_{2}\right)-\psi_{2, x}^{2}\left(k_{1}, k_{2}, k_{3}\right)=\xi_{3, s}^{3}-\xi_{2, s}^{2}+ \\
\phi_{h}\left(-k_{1}-k_{5}-k_{3}-k_{4}-k_{2}\right)-\phi_{h}\left(-k_{1}-k_{2}-k_{3}\right)+ \\
\phi_{h}\left(k_{5}\right)-\phi_{h}\left(-k_{4}\right)
\end{array}
$$

Next, $k_{2}, k_{3}$ and $k_{4}$ have to be fixed to particular frequencies $\alpha, \beta$ and $\gamma(0 \leq \alpha, \beta, \gamma<N)$, respectively, chosen using both the $1 \mathrm{D}$ and $2 \mathrm{D}$ frequency content criteria defined in section IIIC. Summing (14) over all the discrete frequencies $k_{1}\left(0 \leq k_{1}<\right.$ $N-1)$, it can be easy shown that, for every $k_{5}\left(0 \leq k_{5}<N\right)$ :

$$
\begin{array}{r}
\sum_{k_{1}=0}^{N-1} \psi_{3, x}^{3}\left(k_{1}, k_{5}, \beta, \gamma, \alpha\right)-\psi_{2, x}^{2}\left(k_{1}, \alpha, \beta\right)= \\
N\left(\phi_{h}\left(k_{5}\right)-\phi_{h}(-\gamma)+\xi_{3, s}^{3}-\xi_{2, s}^{2}\right)
\end{array}
$$

Therefore, the phase response $\phi_{h}$ could be estimated from the previous equation. However, as it was said in section III-A, the output polyspectrum phase has to be estimated through its principal value. So, in order to obtain the true phase up to an additive constant, we must perform an additional step of phase unwrapping (see problem 2). This problem can be resolved in several ways, depending on the level where the unwrapping scheme is applied to the output quintuspectrum and trispectrum phases. For the sake of convenience, in this section we only present two different approaches. The first one consists of applying a 2D phase unwrapping method to function $\tilde{\psi}_{3, x}^{3}(., ., \beta, \gamma, \alpha)$ defined from $\tilde{\psi}_{3, x}^{3}$ by fixing some frequencies to $\beta, \gamma$ and $\alpha$ respectively. In fact, since $\tilde{\psi}_{3, x}^{3}$ is a function of five variables, $\tilde{\psi}_{3, x}^{3}(., ., \beta, \gamma, \alpha)$ is obtained from $\tilde{\psi}_{3, x}^{3}$ by fixing its three last variables, namely $k_{3}, k_{4}$ and $k_{2}$ (see equation (14)), to $\beta, \gamma$ and $\alpha$ respectively. So the unwrapped function $\tilde{\psi}_{3, x}^{3, u}(., ., \beta, \gamma, \alpha)$ and $\tilde{\psi}_{2, x}^{2}(., \alpha, \beta)$ (where $\tilde{\psi}_{2, x}^{2}(., \alpha, \beta)$ is obtained from $\tilde{\psi}_{2, x}^{2}$ by fixing $k_{2}$ and $k_{3}$ to $\alpha$ and $\beta$, respectively) are subtracted and then they are summed over all the frequencies $k_{1}\left(0 \leq k_{1}<N-1\right)$. Finally, the result is divided by $N$ in order to estimate $\phi_{h}$ up to an additive constant. The second approach consists of i) summing up the function $\tilde{\psi}_{3, x}^{3}(., ., \beta, \gamma, \alpha)-\tilde{\psi}_{2, x}^{2}(., \alpha, \beta)$ with respect to its first variable, $k_{1}$, over all the discrete values belonging to $\{0,1, \ldots, N-1\}$, ii) unwrapping the result using a $1 \mathrm{D}$ phase unwrapping process, and iii) dividing the result by $N$. In the sequel, these two methods will be referred to as the $(4,6)$ $\mathrm{PEP}_{2 \mathrm{D}}$ and $(4,6)-\mathrm{PEP}_{1 \mathrm{D}}$ algorithms, respectively.

Moreover, as explained in the previous section, one should be cautious about the choice of $\gamma$. Indeed, if the taps of the impulse response are in the complex field, a non-zero value has to be chosen for $\gamma$ in order to be able to estimate $\phi_{h}(0)$.

\section{Toward a 2D frequency content criterion}

One of the particularities of the PEP methods with regard to the others is the need of a "good" 2D polyspectrum slice. Therefore, we decided to extend the criterion proposed by Pozidis et al. [21, eq. 25] for the selection of the best 1D slice.
More precisely, in order to select the appropriate $q$-th order $(q>3)$ 2D slice, we propose to choose the set $\left(\alpha_{1}, \ldots, \alpha_{q-3}\right)$ which maximizes the following criterion:

$F C_{2 \mathrm{D}}\left(\alpha_{1}, \ldots, \alpha_{q-3}\right)=\frac{1}{N^{2}} \sum_{k_{1}=0}^{N-1} \sum_{k_{2}=0}^{N-1}\left|\Gamma_{r, x}^{q-r}\left(k_{1}, k_{2}, \alpha_{1}, \ldots, \alpha_{q-3}\right)\right|$

This procedure implies the estimation of all the $q$-th order 2D slices. Another way, less expensive in terms of computational cost, consists of i) choosing the frequency index $\bar{\alpha}$ which maximizes the modulus of the power spectrum $\Gamma_{1, x}^{1}$ and ii) fixing the set $\left(\alpha_{1}, \ldots, \alpha_{q-3}\right)$ to $(\bar{\alpha}, \ldots, \bar{\alpha})$. This idea was first suggested by Pozidis et al. [21] in order to select the best $q$-th order 1D slice.

\section{AN ANALYSIS OF ASYMPTOTIC CONSISTENCY OF THE PEP APPROACH}

We provide, in this section, an analysis of the asymptotic behavior of the $4-\mathrm{PEP}_{2 \mathrm{D}}$ and $(4,6)-\mathrm{PEP}_{2 \mathrm{D}}$ methods when Flynn's algorithm is used in order to achieve a $2 \mathrm{D}$ phase unwrapping [8]. Indeed, Flynn's method is one of the most robust algorithms with respect to false discontinuities [8], which could appear in the wrapped phase because, for instance, of a poor frequency sampling of the corresponding polyspectrum.

\section{A. Preliminary results}

As a first result, let's recall the following lemma [4]:

Lemma 1: Let $\hat{\Gamma}_{r, x}^{q-r}$ be the estimate of the $q$-th order $(q \geq 2)$ spectrum, $\Gamma_{r, x}^{q-r}$, of a stationary-ergodic process $\{x(m)\}_{m \in \mathbb{Z}}$, defined as a weighted smoothing of the q-th order periodogram. By properly choosing the weighting sequence with increasing sample size, and under suitable regularity conditions, the estimate $\hat{\Gamma}_{r, x}^{q-r}$ is asymptotically unbiased and consistent.

Now, what happens as far as the wrapped phase, $\hat{\tilde{\psi}}_{r, x}^{q-r}$, of $\hat{\Gamma}_{r, x}^{q-r}$ is concerned? Does it converge in probability to the wrapped phase, $\tilde{\psi}_{r, x}^{q-r}$, of $\Gamma_{r, x}^{q-r}$ ? If it does, is it asymptotically unbiased and consistent? The answer ensues from the following results:

Theorem 1: Under the assumptions of lemma 1, the wrapped phase, $\hat{\tilde{\psi}}_{r, x}^{q-r}$, of $\hat{\Gamma}_{r, x}^{q-r}$ converges in probability to the wrapped phase, $\tilde{\psi}_{r, x}^{q-r}$, of $\Gamma_{r, x}^{q-r}$.

A proof is given in appendix VII-A. The following corollary ensues from theorem 1.

Corollary 1: Under the assumptions of lemma 1, the wrapped phase, $\hat{\tilde{\psi}}_{r, x}^{q-r}$, of $\hat{\Gamma}_{r, x}^{q-r}$ is asymptotically unbiased and consistent.

The proof is then straightforward since the phase estimation error is bounded. Before analyzing the asymptotic properties of the estimate $N \hat{\tilde{\phi}}_{h}$ of $N \phi_{h}$ when $4-\mathrm{PEP}_{2 \mathrm{D}}$ and $(4,6)-\mathrm{PEP}_{2 \mathrm{D}}$ are used, let's consider the following result:

Theorem 2: Let $\boldsymbol{\alpha}=\left(\alpha_{1}, \ldots, \alpha_{q-3}\right), \tilde{\psi}_{r, x}^{q-r, u}(., ., \boldsymbol{\alpha})$ and $\hat{\tilde{\psi}}_{r, x}^{q-r, u}(., ., \boldsymbol{\alpha})$ be a $q-3$-tuple of $\{0,1, \ldots, N-1\}^{q-3}$, the unwrapped phases computed by applying Flynn's method to the 
wrapped phases $\tilde{\psi}_{r, x}^{q-r}(., ., \boldsymbol{\alpha})$ and $\hat{\tilde{\psi}}_{r, x}^{q-r}(., ., \boldsymbol{\alpha})$, respectively. Under the assumptions of lemma 1 , the estimate $\hat{\tilde{\psi}}_{r, x}^{q-r, u}(., ., \boldsymbol{\alpha})$ of $\tilde{\psi}_{r, x}^{q-r, u}(., ., \boldsymbol{\alpha})$ is asymptotically unbiased and consistent.

A proof is given in appendix VII-B.

Now let $W(k)$ and $V\left(k, k^{\prime}\right)$ be random variables defined by $W(k)=\hat{\tilde{\psi}}_{r, x}^{q-r}(k, \boldsymbol{\beta})-\tilde{\psi}_{r, x}^{q-r}(k, \boldsymbol{\beta})$ and $V\left(k, k^{\prime}\right)=$ $\hat{\tilde{\psi}}_{r, x}^{q-r, u}\left(k, k^{\prime}, \boldsymbol{\alpha}\right)-\tilde{\psi}_{r, x}^{q-r, u}\left(k, k^{\prime}, \boldsymbol{\alpha}\right)$, respectively, where $(k, k) \in$ $\{0, \ldots, N-1\}^{2}, \boldsymbol{\beta}=\left(\beta_{1}, \ldots, \beta_{q-2}\right) \in\{0, \ldots, N-1\}^{q-2}$ and $\boldsymbol{\alpha}=\left(\alpha_{1}, \ldots, \alpha_{q-3}\right) \in\{0, \ldots, N-1\}^{q-3}$. Next, let $L^{2}(\Omega, \mathcal{T}, \mathcal{P})$ be the Hilbert space of second order random variables. Since $W(k)$ and $V\left(k, k^{\prime}\right)$ are elements of $L^{2}(\Omega, \mathcal{T}, \mathcal{P})$, using corollary 1, theorem 2 and Schwarz's inequality we get the following proposition.

Proposition 1: Under the assumptions of lemma 1, $\mathbb{E}\left[W(k) W\left(k^{\prime}\right)\right], \mathbb{E}\left[V\left(k, k^{\prime}\right) V\left(k^{\prime \prime}, k^{\prime}\right)\right]$ and $\mathbb{E}\left[V\left(k, k^{\prime}\right) W\left(k^{\prime \prime}\right)\right]$ converge to zero, where $\mathbb{E}$ denotes the mathematical expectation operator.

\section{B. About the 4-PEP $2 \mathrm{D}$ and $(4,6)-P E P_{2 \mathrm{D}}$ methods}

According to equation (12) and using the notations of the previous subsection, for every $k_{1}$ belonging to $\{0, \ldots, N-1\}$, we get:

$$
N\left(\hat{\tilde{\phi}}_{h}\left(k_{1}\right)-\tilde{\phi}_{h}\left(k_{1}\right)\right)=\sum_{k_{2}=0}^{N-1} V\left(k_{1}, k_{2}\right)
$$

Thus we deduce from (17), theorem 2 and the linearity of the mathematical expectation that the estimated phase, $\hat{\tilde{\phi}}_{h}$, obtained by the $4-\mathrm{PEP}_{2 \mathrm{D}}$ method is asymptotically unbiased. The same result is also true for the $(4,6)-\mathrm{PEP}_{2 \mathrm{D}}$ algorithm. In fact, the following equation can be easily derived from sections III-B and IV-A:

$$
N\left(\hat{\tilde{\phi}}_{h}\left(k_{5}\right)-\tilde{\phi}_{h}\left(k_{5}\right)\right)=\sum_{k_{1}=0}^{N-1}\left(V\left(k_{1}, k_{5}\right)-W\left(k_{1}\right)\right)
$$

The asymptotically unbiasedness of $\hat{\tilde{\phi}}_{h}$ is then directly deduced from (18), corollary 1 , theorem 2 and the linearity of the mathematical expectation.

Now the variances of $\hat{\tilde{\phi}}_{h}$ associated to the $4-\mathrm{PEP}_{2 \mathrm{D}}$ and $(4,6)-\mathrm{PEP}_{2 \mathrm{D}}$ methods are given by:

$$
\begin{aligned}
& N^{2} \mathbb{E}\left[\left(\hat{\tilde{\phi}}_{h}\left(k_{1}\right)-\tilde{\phi}_{h}\left(k_{1}\right)\right)^{2}\right]= \\
& \quad \sum_{k_{2}=0}^{N-1} \sum_{k_{2}^{\prime}=0}^{N-1} \mathbb{E}\left[V\left(k_{1}, k_{2}\right) V\left(k_{1}, k_{2}^{\prime}\right)\right]
\end{aligned}
$$

and

$$
\begin{aligned}
& N^{2} \mathbb{E}\left[\left(\hat{\tilde{\phi}}_{h}\left(k_{5}\right)-\tilde{\phi}_{h}\left(k_{5}\right)\right)^{2}\right]= \\
& \sum_{k_{1}=0 k_{1}^{\prime}=0}^{N-1} \sum^{N-1}\left(\mathbb{E}\left[V\left(k_{1}, k_{5}\right) V\left(k_{1}^{\prime}, k_{5}\right)\right]-\mathbb{E}\left[V\left(k_{1}, k_{5}\right) W\left(k_{1}^{\prime}\right)\right]+\right. \\
& \left.\mathbb{E}\left[W\left(k_{1}\right) W\left(k_{1}^{\prime}\right)\right]-\mathbb{E}\left[W\left(k_{1}\right) V\left(k_{1}^{\prime}, k_{5}\right)\right]\right)
\end{aligned}
$$

respectively. Then, using proposition 1 it appears that both previous equations converge to zero, which shows the consistency of the $4-\mathrm{PEP}_{2 \mathrm{D}}$ and $(4,6)-\mathrm{PEP}_{2 \mathrm{D}}$ algorithms.

\section{COMPUTER SIMULATIONS}

The objective of this section is twofold: i) to demonstrate the performances of the proposed PEP algorithms, applied to band-limited systems, comparing them with those of some efficient phase retrieval techniques, referred to as Petro/Pozi [20] and $q$-Pozi/Petro [21] ( $q=3$ and $q=4$ when the output bispectrum and trispectrum are used respectively) and ii) to show that the PEP methods are more robust to a wrong choice of the polyspectrum slices used in the reconstruction procedure compared to methods quoted previously. Note that, although the Pozi/Petro approach [21] allows for complete system reconstruction, we only evaluate its ability in recovering the system phase. Moreover, we decided to show the performances of the $\mathrm{PEP}_{2 \mathrm{D}}$ methods, that is, the PEP algorithms which use a $2 \mathrm{D}$ unwrapping scheme, since simulations proved their superiority over the other PEP approaches. Recall that Flynn's minimum discontinuity method [8] was used, as far as the 2D unwrapping processing is concerned. D.C. Ghiglia et al. [8, pp.151-177] show that this finds a solution that minimizes the discontinuities. The algorithm achieves this goal by using a tree-growing approach that traces paths of discontinuities in the phase, detects the paths that form loops, and adds multiples of $2 \pi$ to the phase values enclosed by the loops to minimize the discontinuities. This process is performed iteratively until no more loops are detected. The process is guaranteed to converge to a "minimum discontinuity" solution.

So, four computer experiments are presented in the following subsections in order to compare the $\mathrm{PEP}_{2 \mathrm{D}}$ methods with the Petro/Pozi, 3-Pozi/Petro and 4-Pozi/Petro algorithms. In each experiment, two stationary processes were generated using two non-minimum phase bandlimited systems defined by:

$$
\begin{aligned}
\forall m \in\{-9, \cdots, 6\}, & h_{1}(m)=0.77^{\left|\frac{m}{2}\right|} \cos (0.49 \pi m) \\
& +0.8(0.65)^{\left|\frac{m}{2}\right|} \sin \left(0.38 \pi+\frac{\pi}{5}\right)
\end{aligned}
$$

and:

$$
\begin{array}{r}
H_{2}(z)=1-1.25 z^{-1}+1.75 z^{-2}-5.25 z^{-3}-12.5 z^{-4} \\
+18 z^{-5}+9 z^{-6}-2.86 \times 10^{-6} z^{-7}+5.25 z^{-8} \\
+0.75 z^{-9}+1.75 z^{-10}
\end{array}
$$

where $h_{1}$ and $H_{2}$ represent the impulse response of a bandpass filter and the transfert function of a proakis-a channel, respectively. It should be noted that the discrete-time proakisa channel is typical of the response of a good quality telephone channel [22]. Next an additive zero-mean white gaussian noise was added to the outputs. The noisy sequence is then divided to records of 256 samples, and an indirect method is used to estimate the polyspectra, with a DFT size of 64 samples. As a performance index of the phase estimation methods, we used the Normalized Mean-Squared Error (NMSE) criterion [5, eq. 53] between the true channel impulse response and its estimated impulse response. Note that the estimated impulse response was computed in time-domain using the true filter magnitude combined with the recovered phase. 


\section{A. Data length effects}

In this experiment, we set the Signal to Noise Ratio (SNR) to $15 \mathrm{~dB}$ and varied the data length. Two different studies were then conducted. The first one relates to non-symmetric input sources (we used a zero-mean exponentially distributed i.i.d. sequence) and the second one deals with symmetrically distributed sources (a Binary Phase Shift Keying (BPSK) source in baseband with a square transmit filter and a symbol rate equal to the sample rate was then used). Consequently, since the i.i.d. exponential sequence has a non-zero skewness, the first study allows to compare the performance of the $3-\mathrm{PEP}_{2 \mathrm{D}}$, $(3,4)-\mathrm{PEP}_{2 \mathrm{D}}$, Petro/Pozi and the 3-Pozi/Petro algorithms. On the other hand, since the BPSK signal is symmetrically distributed, the trispectrum $(q=4)$ of the observations was used in the second study in order to compare the performances of the $4-\mathrm{PEP}_{2 \mathrm{D}}$ and 4 -Pozi/Petro techniques.

Figures 1(a), 1(b) and figures 1(c), 1(d) display the obtained results corresponding to the exponentially distributed i.i.d sequence and to the BPSK source, respectively. In both cases, the variations of the NMSE criterion for both impulse responses $h_{1}$ and $h_{2}$ as a function of the data length show clearly that the PEP methods perform better than the other methods, except in figure $1(\mathrm{~b})$ where the performance of the $(3,4)-\mathrm{PEP}_{2 \mathrm{D}}$ for $h_{2}$, Petro/Pozi and 3-Pozi/Petro methods are equivalent.

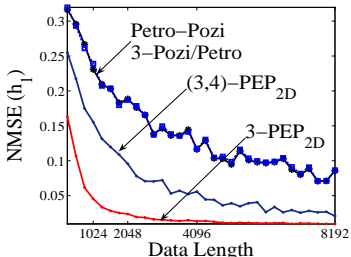

(a)

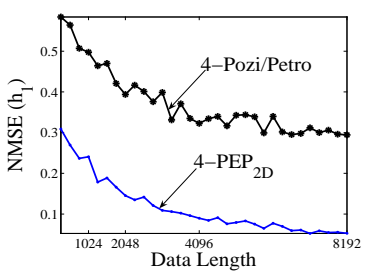

(c)

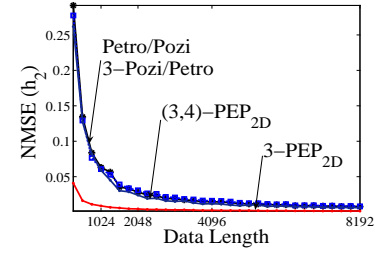

(b)

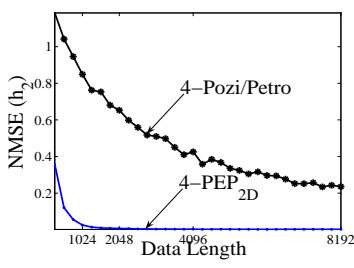

(d)
Fig. 1. NMSE as a function of the data length: (a) and (b) for an exponential distribution and (c) and (d) for a BPSK.

\section{B. Signal to noise ratio effects}

We generated 2048 samples of data and varied the SNR. Then the performance of the six methods cited in V-A were compared again. As in the previous experiment, two kinds of results were obtained: those associated with a zero-mean exponentially distributed i.i.d. sequence, plotted in figures 2(a) and 2(b) and those dealing with a BPSK, shown in figures 2(c) and 2(d). It appears in figures 2(a) and 2(b) that the $\mathrm{PEP}_{2 \mathrm{D}}$ methods lead to better results, especially for low SNR values. The superiority of the $\mathrm{PEP}_{2 \mathrm{D}}$ approach is even more obvious in figures 2(c) and 2(d), whatever the SNR value.

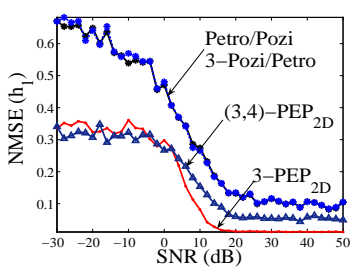

(a)

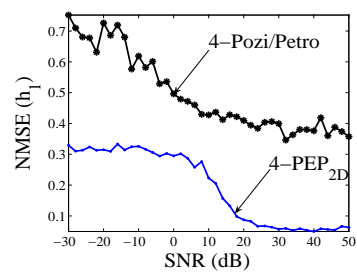

(c)

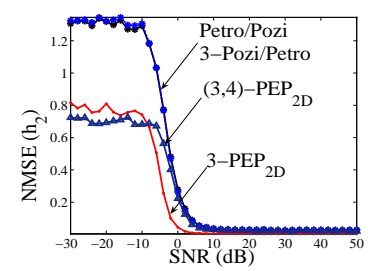

(b)

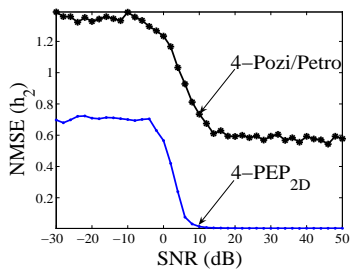

(d)
Fig. 2. NMSE as a function of the SNR: (a) and (b) for an exponential distribution and (c) and (d) a BPSK.

\section{C. $1 D$ or $2 D$ slice selection effects}

In this experiment, the data length and the SNR were both fixed to 2048 samples and $15 \mathrm{~dB}$, respectively and two different kinds of input sequence, namely the zero-mean exponentially distributed i.i.d. signal and the BPSK source, were considered again. The NMSE of the output of the Petro/Pozi, 3-Pozi/Petro, 4-Pozi/Petro, 4-PEP $2 \mathrm{D}$, (3,4)-PEP $2 \mathrm{D}$ and the $(4,6)-\mathrm{PEP}_{2 \mathrm{D}}$ methods were computed as a function of the used polyspectrum slice $\bar{\alpha}(\bar{\alpha} \in\{1, \ldots, N-1\})$ introduced in section III-C. The results are presented, in figures 3(a) and 3(b) for an exponentially distributed i.i.d. sequence and in figures 3(c) and 3(d) for the BPSK source, in parallel with the Magnitude Response (MR) of the corresponding filter. Note that, in order to improve the readability of the results, the MR was normalized with respect to the maximum value of NMSE obtained at the output of the different algorithms. Clearly the $(3,4)-\mathrm{PEP}_{2 \mathrm{D}}$ and $(4,6)-\mathrm{PEP}_{2 \mathrm{D}}$ methods seem to offer a much greater flexibility with respect to the polyspectrum slice selection and they seem to be more robust when the chosen slice corresponds to a region where the noise contribution to the polyspectrum output dominates the input signal contribution.

\section{CONCLUSION}

We propose in this paper a new family of methods, called PEP (Phase Estimation using Polyspectrum slices) methods, in order to solve the phase estimation problem for nonminimum phase systems. These methods, exploit only 1D and 2D slices of higher order spectra. They can be divided in two classes: the $q$-PEP ( $q \geq 3$ ) methods, which use one particular 2D slice of the $q$-th order spectrum, and the $\left(q_{1}, q_{2}\right)$ PEP $\left(q_{2}>q_{1} \geq 3\right)$ methods, based on the joint exploitation of one special 1D slice of the $q_{1}$-th order spectrum and one particular $2 \mathrm{D}$ slice of the $q_{2}$-th order spectrum. There are some important differences between our methods and those proposed by Petropulu et al. [20] and by Pozidis et al. [21]. Indeed, while the Petro/Pozi [20] is a recursive method and the Pozi/Petro [21] can be considered as a block method; our 


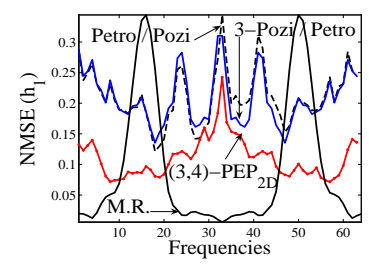

(a)

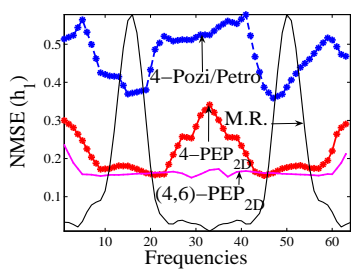

(c)

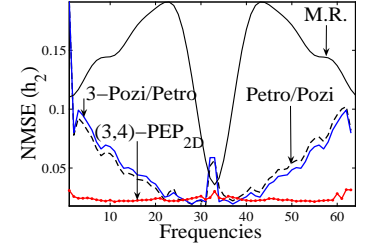

(b)

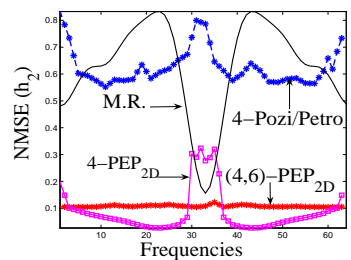

(d)
Fig. 3. NMSE as a function of the frequency slice, with a data length of 2048 samples and SNR=15 dB: (a) and (b) for an exponential distribution and (c) and (d) for a BPSK

methods estimate each phase value independently from the others. Contrary to Petro/Pozi and Pozi/Petro which are based on two 1D slices of the q-th order spectrum, the q-PEP approaches exploit one 2D slice q-th order spectrum. Regarding the $\left(q_{1}, q_{2}\right)$-PEP methods, its originality is the simultaneous exploitation of one 1D slice of $q_{1}$-th order spectrum and one 2D slice of $q_{2}$-th order spectrum. This joint exploitation of one 1D slice and one 2D slice has never been proposed in literature. An important result of this joint exploitation of two distinct q-th order spectra is the flexibility of the $\left(q_{1}, q_{2}\right)$-PEP methods regarding the frequency slice selection compared to the classical methods. It is also shown in this paper that the PEP methods are easy to implement and they are appropriate in any kind of system, whether real or complex. In addition, the recovered phases are shown to be asymptotically consistent. The good behavior of the $\mathrm{PEP}_{2 \mathrm{D}}$ is confirmed through several computer simulations. Indeed, for band-limited systems, the $\mathrm{PEP}_{2 \mathrm{D}}$ methods provide superior performance, as compared with the classical algorithms, for both symmetrically and nonsymmetrically distributed sources.

\section{APPENDIX}

\section{A. Proof of theorem 1}

For the sake of readability and clarity, we omitted the " $q-1$ "tuple $\left(k_{1}, \ldots, k_{q-1}\right)$ in the sequel, that is to say, the point $\left(k_{1}, \ldots, k_{q-1}\right)$ where the function $f$ is evaluated is omitted and value $f\left(k_{1}, \ldots, k_{q-1}\right)$ is simply denoted by $f$. Indeed, the following proof is valid for any point $\left(k_{1}, \ldots, k_{q-1}\right) \in$ $\{0,1, \ldots, N-1\}^{q-1}$. Then, let $a, b, \hat{a}, \hat{b}, \delta a$ and $\delta b$ be the real part of $\Gamma_{r, x}^{q-r}$, the imaginary part of $\Gamma_{r, x}^{q-r}$, the real part of $\hat{\Gamma}_{r, x}^{q-r}$, the imaginary part of $\hat{\Gamma}_{r, x}^{q-r}$, the difference $a-\hat{a}$ and the difference $b-\hat{b}$, respectively. Let's recall that $M$ denotes the number of samples used to estimate $\Gamma_{r_{2} x}^{q-r}$.

Now, let's consider the wrapped phase $\tilde{\psi}_{r, x}^{q-r}$. Since it may be close to $\pm \pi$ and since its estimate $\hat{\tilde{\psi}}_{r, x}^{q-r}$ is also a wrapped phase, $\hat{\tilde{\psi}}_{r, x}^{q-r}$ can be decomposed as $\tilde{\psi}_{r, x}^{q-r}+\delta \tilde{\psi}+2 \pi \eta$ where $\delta \tilde{\psi}$ is the smallest phase error such that $\eta$ belongs to $\{-1,0,1\}$. The purpose of this appendix consists then in showing that $\delta \tilde{\psi}$ converges in probability to zero.

For evrey non-zero positive real $\epsilon$, it exists at least one nonzero positive rreal $\epsilon^{\prime}$ such that $|\delta a|<\epsilon^{\prime}$ and $|\delta b|<\epsilon^{\prime}$ implies $|\delta \psi|<\epsilon$. Indeed, it suffices to take $\epsilon^{\prime}=\sqrt{2} \sqrt{a^{2}+b^{2}}|\sin (\epsilon)|$ as shown in figure 4 . So for every non-zero positive real $\epsilon$, it exists at least one non-zero positive real $\epsilon^{\prime}$ such that:

$$
P\left(|\delta a|<\epsilon^{\prime} \text { and }|\delta b|<\epsilon^{\prime}\right) \leq P(|\delta \psi|<\epsilon)
$$

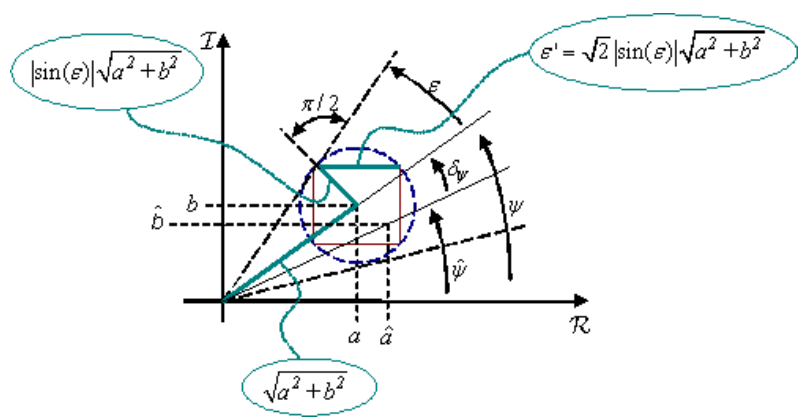

Fig. 4. A geometric proof in order to relate the real number $\epsilon^{\prime}$ to $\epsilon$

Now, let's recall that convergence in mean square implies convergence in probability according to Tchebycheff inequality [18]. Consequently, since $\hat{\Gamma}_{r, x}^{q-r}$ is asymptotically unbiased and consistent according to lemma 1 , both $\delta a$ and $\delta b$ converge in probability to zero.

Therefore, for every non-zero positive real $\epsilon^{\prime}$, the left term of the inequality above converges to one when $M$ goes to infinity. Hence the convergence of $P(|\delta \psi|<\epsilon)$ to one using the same inequality.

\section{B. Proof of theorem 2}

Let $I$ and $\hat{I}$ be the phase jump functions computed by Flynn's method from the wrapped phases $\tilde{\psi}_{r, x}^{q-r}(., ., \boldsymbol{\alpha})$ and $\hat{\tilde{\psi}}_{r, x}^{q-r}(., ., \boldsymbol{\alpha})$, respectively, such that, for every $\left(k_{1}, k_{2}, \boldsymbol{\alpha}\right)$ of $\{0,1, \ldots, N-1\}^{2}$ :

$$
\tilde{\psi}_{r, x}^{q-r, u}\left(k_{1}, k_{2}, \boldsymbol{\alpha}\right)=\tilde{\psi}_{r, x}^{q-r}\left(k_{1}, k_{2}, \boldsymbol{\alpha}\right)+2 \pi I\left(k_{1}, k_{2}, \boldsymbol{\alpha}\right)
$$

and:

$$
\hat{\tilde{\psi}}_{r, x}^{q-r, u}\left(k_{1}, k_{2}, \boldsymbol{\alpha}\right)=\hat{\tilde{\psi}}_{r, x}^{q-r}\left(k_{1}, k_{2}, \boldsymbol{\alpha}\right)+2 \pi \hat{I}\left(k_{1}, k_{2}, \boldsymbol{\alpha}\right)
$$

with, according to the notations of appendix VII-A:

$$
\begin{array}{r}
\hat{\tilde{\psi}}_{r, x}^{q-r}\left(k_{1}, k_{2}, \boldsymbol{\alpha}\right)=\tilde{\psi}_{r, x}^{q-r}\left(k_{1}, k_{2}, \boldsymbol{\alpha}\right)+\delta \tilde{\psi}\left(k_{1}, k_{2}, \boldsymbol{\alpha}\right) \\
+2 \pi \eta\left(k_{1}, k_{2}, \boldsymbol{\alpha}\right)
\end{array}
$$

We know that Flynn's method finds the phase jump function $I$ of (21) by minimizing, with respect to $I$, the following global 
criterion:

$$
\begin{aligned}
& \Upsilon(I)= \\
& \quad \sum_{k_{1}, k_{2}}\left|\operatorname{Int}\left(\frac{\tilde{\psi}_{r, x}^{q-r, u}\left(k_{1}, k_{2}, \boldsymbol{\alpha}\right)-\tilde{\psi}_{r, x}^{q-r, u}\left(k_{1}+1, k_{2}, \boldsymbol{\alpha}\right)}{2 \pi}\right)\right| \\
& +\sum_{k_{1}, k_{2}}\left|\operatorname{Int}\left(\frac{\tilde{\psi}_{r, x}^{q-r, u}\left(k_{1}, k_{2}, \boldsymbol{\alpha}\right)-\tilde{\psi}_{r, x}^{q-r, u}\left(k_{1}, k_{2}+1, \boldsymbol{\alpha}\right)}{2 \pi}\right)\right|
\end{aligned}
$$

where Int(.) denotes the function that rounds to the nearest integer. Inserting (21) into (24), we get the following global criterion:

$$
\Upsilon(I)=\sum_{k_{1}, k_{2}}\left|A\left(k_{1}, k_{2}, \boldsymbol{\alpha}\right)\right|+\sum_{k_{1}, k_{2}}\left|B\left(k_{1}, k_{2}, \boldsymbol{\alpha}\right)\right|
$$

with:

$$
\begin{array}{r}
\left.A\left(k_{1}, k_{2}, \boldsymbol{\alpha}\right)=\left(I\left(k_{1}, k_{2}, \boldsymbol{\alpha}\right)\right)-I\left(k_{1}+1, k_{2}, \boldsymbol{\alpha}\right)\right)+ \\
\operatorname{Int}\left(\tilde{\psi}\left(k_{1}, k_{2}, \boldsymbol{\alpha}\right)-\tilde{\psi}\left(k_{1}+1, k_{2}, \boldsymbol{\alpha}\right) / 2 \pi\right) \\
\left.B\left(k_{1}, k_{2}, \boldsymbol{\alpha}\right)=\left(I\left(k_{1}, k_{2}, \boldsymbol{\alpha}\right)\right)-I\left(k_{1}, k_{2}+1, \boldsymbol{\alpha}\right)\right)+ \\
\operatorname{Int}\left(\tilde{\psi}\left(k_{1}, k_{2}, \boldsymbol{\alpha}\right)-\tilde{\psi}\left(k_{1}, k_{2}+1, \boldsymbol{\alpha}\right) / 2 \pi\right)
\end{array}
$$

Now, what does Flynn's method give when it minimizes, with respect to $\hat{I}$ of (22), the following global criterion, that is to say, when $\tilde{\psi}_{r, x}^{q-r}$ is estimated by $\tilde{\tilde{\psi}}_{r, x}^{q-r}$ ?

$$
\begin{aligned}
& \Upsilon(\hat{I})= \\
& \quad \sum_{k_{1}, k_{2}}\left|\operatorname{Int}\left(\frac{\hat{\tilde{\psi}}_{r, x}^{q-r, u}\left(k_{1}, k_{2}, \boldsymbol{\alpha}\right)-\hat{\tilde{\psi}}_{r, x}^{q-r, u}\left(k_{1}+1, k_{2}, \boldsymbol{\alpha}\right)}{2 \pi}\right)\right| \\
& +\sum_{k_{1}, k_{2}}\left|\operatorname{Int}\left(\frac{\hat{\tilde{\psi}}_{r, x}^{q-r, u}\left(k_{1}, k_{2}, \boldsymbol{\alpha}\right)-\hat{\tilde{\psi}}_{r, x}^{q-r, u}\left(k_{1}, k_{2}+1, \boldsymbol{\alpha}\right)}{2 \pi}\right)\right|
\end{aligned}
$$

Inserting (22) and (23) into (27), we get:

$$
\Upsilon(\hat{I})=\sum_{k_{1}, k_{2}}\left|\hat{A}\left(k_{1}, k_{2}, \boldsymbol{\alpha}\right)\right|+\sum_{k_{1}, k_{2}}\left|\hat{B}\left(k_{1}, k_{2}, \boldsymbol{\alpha}\right)\right|
$$

with:

$$
\begin{aligned}
& \hat{A}\left(k_{1}, k_{2}, \boldsymbol{\alpha}\right)=\left(\eta\left(k_{1}, k_{2}, \boldsymbol{\alpha}\right)+\hat{I}\left(k_{1}, k_{2}, \boldsymbol{\alpha}\right)\right. \\
& \left.-\eta\left(k_{1}+1, k_{2}, \boldsymbol{\alpha}\right)-\tilde{I}\left(k_{1}+1, k_{2}, \boldsymbol{\alpha}\right)\right)+\operatorname{Int}\left(\tilde{\psi}\left(k_{1}, k_{2}, \boldsymbol{\alpha}\right)\right. \\
& \left.+\delta \tilde{\psi}\left(k_{1}, k_{2}, \boldsymbol{\alpha}\right)-\tilde{\psi}\left(k_{1}+1, k_{2}, \boldsymbol{\alpha}\right)-\delta \tilde{\psi}\left(k_{1}+1, k_{2}, \boldsymbol{\alpha}\right) / 2 \pi\right) \\
& \hat{B}\left(k_{1}, k_{2}, \boldsymbol{\alpha}\right)=\left(\eta\left(k_{1}, k_{2}, \boldsymbol{\alpha}\right)+\hat{I}\left(k_{1}, k_{2}, \boldsymbol{\alpha}\right)\right. \\
& \left.-\eta\left(k_{1}, k_{2}+1, \boldsymbol{\alpha}\right)-\hat{I}\left(k_{1}, k_{2}+1, \boldsymbol{\alpha}\right)\right)+\operatorname{Int}\left(\tilde{\psi}\left(k_{1}, k_{2}, \boldsymbol{\alpha}\right)\right. \\
& \left.+\delta \tilde{\psi}\left(k_{1}, k_{2}, \boldsymbol{\alpha}\right)-\tilde{\psi}\left(k_{1}, k_{2}+1, \boldsymbol{\alpha}\right)-\delta \tilde{\psi}\left(k_{1}, k_{2}+1, \boldsymbol{\alpha}\right) / 2 \pi\right)
\end{aligned}
$$

In fact, we deduce from theorem 1 that both variables $\delta \tilde{\psi}\left(k_{1}, k_{2}, \boldsymbol{\alpha}\right)-\delta \tilde{\psi}\left(k_{1}+1, k_{2}, \boldsymbol{\alpha}\right)$ and $\delta \tilde{\psi}\left(k_{1}, k_{2}, \boldsymbol{\alpha}\right)-$ $\delta \tilde{\psi}\left(k_{1}, k_{2}+1, \boldsymbol{\alpha}\right)$ converge in probability to zero. Consequently, if we assume that both variables $\mid \tilde{\psi}\left(k_{1}, k_{2}, \boldsymbol{\alpha}\right)-$ $\tilde{\psi}\left(k_{1}+1, k_{2}, \boldsymbol{\alpha}\right) \mid$ and $\left|\tilde{\psi}\left(k_{1}, k_{2}, \boldsymbol{\alpha}\right)-\tilde{\psi}\left(k_{1}, k_{2}+1, \boldsymbol{\alpha}\right)\right|$ are not exactly equal to $\pi$, we can asymptotically neglect the effect of the phase error terms $\delta \tilde{\psi}$ in equation (29), which leads asymptotically:

$$
\begin{aligned}
\hat{A}\left(k_{1}, k_{2}, \boldsymbol{\alpha}\right)=( & \eta\left(k_{1}, k_{2}, \boldsymbol{\alpha}\right)+\hat{I}\left(k_{1}, k_{2}, \boldsymbol{\alpha}\right) \\
& \left.-\eta\left(k_{1}+1, k_{2}, \boldsymbol{\alpha}\right)-\hat{I}\left(k_{1}+1, k_{2}, \boldsymbol{\alpha}\right)\right)+ \\
& \operatorname{Int}\left(\tilde{\psi}\left(k_{1}, k_{2}, \boldsymbol{\alpha}\right)-\tilde{\psi}\left(k_{1}+1, k_{2}, \boldsymbol{\alpha}\right) / 2 \pi\right) \\
\hat{B}\left(k_{1}, k_{2}, \boldsymbol{\alpha}\right)=( & \eta\left(k_{1}, k_{2}, \boldsymbol{\alpha}\right)+\hat{I}\left(k_{1}, k_{2}, \boldsymbol{\alpha}\right) \\
& \left.-\eta\left(k_{1}, k_{2}+1, \boldsymbol{\alpha}\right)-\hat{I}\left(k_{1}, k_{2}+1, \boldsymbol{\alpha}\right)\right)+ \\
& \operatorname{Int}\left(\tilde{\psi}\left(k_{1}, k_{2}, \boldsymbol{\alpha}\right)-\tilde{\psi}\left(k_{1}, k_{2}+1, \boldsymbol{\alpha}\right) / 2 \pi\right)
\end{aligned}
$$

Then, by identifying (26) and (30), we find, for every $\left(k_{1}, k_{2}, \boldsymbol{\alpha}\right)$, that:

$$
\hat{I}\left(k_{1}, k_{2}, \boldsymbol{\alpha}\right)=I\left(k_{1}, k_{2}, \boldsymbol{\alpha}\right)-\eta\left(k_{1}, k_{2}, \boldsymbol{\alpha}\right)
$$

So, inserting (23) and (31) into (22), we have:

$$
\hat{\tilde{\psi}}_{r, x}^{q-r, u}\left(k_{1}, k_{2}, \boldsymbol{\alpha}\right)=\tilde{\psi}_{r, x}^{q-r, u}\left(k_{1}, k_{2}, \boldsymbol{\alpha}\right)+\delta \tilde{\psi}\left(k_{1}, k_{2}, \boldsymbol{\alpha}\right)
$$

According to theorem 1, this last equation clearly shows that $\hat{\tilde{\psi}}_{r, x}^{q-r, u}(., ., \boldsymbol{\alpha})$ is asymptotically unbiased and consistent when Flynn's method is used.

\section{REFERENCES}

[1] H. BARTELT, A. W. LOHMANN, and B. WIRNITZER, "Phase and amplitude recovery from bispectra", Applied Optics, vol. 23, no. 18, pp. 3121-3129, 1984.

[2] A. BENVENISTE, M. GOURSAT and G. RUGET, "Robust identification of nonminimum phase system: Blind ajustement of linear equalizer in data communication", IEEE Trans. on Autom. Control, vol. 25, no. 3, pp. 385-399, 1980.

[3] D. R. BRILLINGER, "The identification of a particular nonlinear times series system", Biometrika, vol. 64, no. 3, pp. 509-515, 1977.

[4] D. R. BRILLINGER and M. ROSENBLATT, "Asymptotic Theory of Estimates of k-th Order Spectra", Proceedings of the National Academy of Sciences of the United States of America, vol. 57, no. 2, pp. 206-210, 1967.

[5] B. CHEN and A. P. PETROPULU, "Frequency domain blind MIMO system identification based on second-and higher-order statistics", IEEE Transactions on Signal Processing, vol. 49, no. 8, pp. 1677-1688, 2001.

[6] R. CUSACK and N. PAPADAKIS, "New robust 3-D phase unwrapping algorithms: application to magnetic field mapping and undistorting echoplanar images", NeuroImage, vol. 16, no. 3, pp.754-764, 2002.

[7] S. A. DIANAT, and M. R RAGHUVEER, "Fast algorithms for bispectral reconstruction of two-dimentional signals", in Proceedings of IEEE International Conference on Acoustics Speech and Signal Processing, pp. 2377-2379, April 1990.

[8] D. C. GHIGLIA and M. D. PRITT, Two-Dimensional Phase Unwrapping: Theory, Algorithms, and Software, Wiley Interscience Publication, New York, 1998.

[9] A. KACHENOURA, L. ALBERA and L. SENHADJI, "The PEP approach: A new family of methods solving the phase estimation problem", in Proc. IEEE Int. Conf. Acoust. Speech Signal Process., May 2006, pp. 628-631.

[10] K. S. LII and M. ROSENBLATT, "Deconvolution and estimation of transfert function phase and coefficients for non-gaussian linear processes", The Annals of Statistics, vol. 10, no. 4, pp. 1195-1208, 1982.

[11] K. S. LII and M. ROSENBLATT, "Asymptotic normality of cumulant spectral estimates", Journal of Theoretical Probability, vol. 3, no. 2, pp. 367-385, 1990.

[12] J. D. MONNIER, "Optical Interferometry in Astronomy", Reports on Progress in Physics, vol. 66, no. 5, pp. 789-857, 2003.

[13] T. MATSUOKA, and T. J. ULRYCH, "Phase estimation using the bispectrum", Proceedings of IEEE, vol. 72, no. 10, pp. 1403-1411, 1984.

[14] P. McCULlaGH, Tensor Methods in Statistics, Chapman and Hall, Monographs on Statistics and Applied Probability, London, 1987.

[15] C. L. NIKIAS, Advances in spectrum analysis and array processing (vol. I), pp. 326-365. Prentice-Hall Advanced Reference Series Engineering, New Jersey, 1991.

[16] C. L. NIKIAS, and A. P. PETROPULU, Higher-Order spectra analysis: a nonlinear signal processing framwork, Prentice-Hall Signal Processing Series, New Jersey, 1993. 
[17] M. RANGOUSSI and G. B. GIANNAKIS, "FIR modeling using logbispectra: Weighted least-squares algorithms and performance analysis", IEEE Trans. Circuits Syst., vol. 38, no. 3, pp. 281-296, March 1991.

[18] A. Papoulis, Probability, random variables and stochastic processes, McGraw-Hill International Editions, Third Edition, New York, 1991.

[19] A. P. PETROPULU, and C. L. NIKIAS, "Signal reconstruction from the phase of bispectrum", IEEE Transactions on Signal Processing, vol. 40, no. 3, pp. 601-610, March 1992.

[20] A. P. PETROPULU and H. POZIDIS, "Phase reconstruction from bispectrum slices", IEEE Transactions on Signal Processing, vol. 46, no. 2, pp. 527-530, February 1998.

[21] H. POZIDIS and A. P. PETROPULU, "System reconstruction based on selected regions of discretized higher order spectra," IEEE Transactions on Signal Processing, vol. 46, no. 12, pp. 3360-3377, December 1998.

[22] J. G. PROAKIS, Digital Communications, 3rd edition, McGraw-Hill, Boston, pp. 616. 1995.

M. RANGOUSSI and G. B. GIANNAKIS, "FIR modeling using logbispectra: Weighted least-squares algorithms and performance analysis", IEEE Transactions On Circuits and Systems, vol. 46, no. 38, pp. 281-296, March 1991.

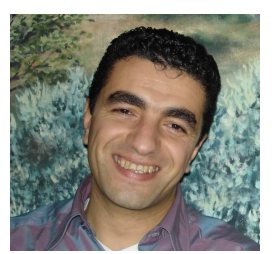

Amar Kachenoura was born in Tizi-Ouzou, Algeria, in 1975. He received the diploma in electronic engineering from M.M.T.O. University, Tizi-Ouzou, Algeria, in 1998 and the DEA degree from Ecole Central de Nantes (ECN), Nantes, France, in July 2002. He received the Ph.D. degree in signal processing from the University of Rennes 1 , Rennes, France, in July 2006. He is currently Post Doctorant in Laboratoire Traitement du Signal et de l'Image (LTSI), INSERM U642-University of Rennes 1, Rennes, France. His research interests focus mainly on biomedical and statistical signal processing, Blind Source Separation (BSS), Independent Component Analysis (ICA) and Brain Computer Interface (BCI) .

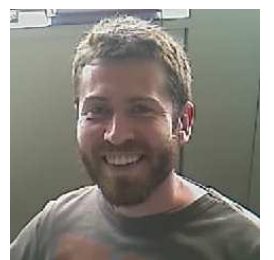

Laurent Albera (M'04) was born in Massy, France, in 1976. He received a D.E.S.S. degree in mathematics and the D.E.A. degree in automatic and signal processing in 2000 and 2001, respectively, from the University of Science of Orsay (Paris XI). Next, he received the Ph.D. degree in sciences from the University of Nice Sophia-Antipolis, France, in 2003. From 2001 to 2004, he was committed for a study within the framework of a Research Contract CIFRE Convention Industrielle de Formation par la Recherche(CIFRE) between Thales Communications (formerly Thomson-CSF), the French National Centre for Scientific Research CNRS, and the University of Nice Sophia-Antipolis. He is now Assistant Professor at the University of Rennes 1 and is affiliated with the INSERM Research Group Laboratoire Traitement du Signal et de 1'Image (LTSI). His research interests include multilinear algebra and high-order statistics in order to perform independent component analysis (ICA) and source localization.

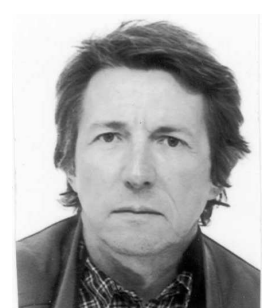

Jean-jacques Bellanger Jean-Jacques Bellanger received the electrical engineering degree in 1971 from the Ecole Nationale d'Ingénieur de Brest, France and the $\mathrm{Ph} . \mathrm{D}$. degree from the university of Rennes 1 in 1997. He is currently associate professor in Signal Processing and Probabilities at the University of Rennes 1 with the department of electrical engineering. He is also affiliated with the INSERM research laboratory "Laboratoire Traitement du Signal et de l'Image, (LTSI)". His research interests are focused processing applied to biomedical signals.

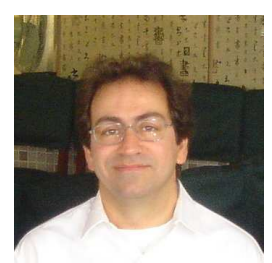

lotfi Senhadji is Professor at the University of Rennes 1 with the department of electrical engineering. He is the head of the INSERM research Laboratory "Laboratoire Traitement du Signal et de l'Image (LTSI)". He received the Ph.D. degree from the University of Rennes 1 , France, in signal processing and telecommunications in 1993. His main research efforts are focused on non-stationary signal processing with particular emphasis on wavelet transforms and time-frequency representations for detection, classification and interpretation of biosignals (Electrocardiography, Electroencephalography, Phonocardiography and NMR Spectroscopy). He has been Investigator of several Grants in the field of Health Technology with public institutions or companies. He has published more than 70 research papers in journals and conferences and he contributed to five handbooks. He is member of the council of the French Society of Biomedical Engineering (SFGBM), the French Alliance for Biomedical Engineering (AGBM) and senior member of IEEE EMBS and SP. 